\title{
Pediatric Nephrology: R N Srivastava and Arvind Bagga (eds)
}

\author{
Published by Jaypee Brothers Medical Publishers (P) Ltd, India, ISBN: 978-93-5025-243-7, \\ Fifth Edition: 2011, Price: Rs. 850
}

\author{
P. K. Pruthi
}

Received: 21 December 2011 / Accepted: 23 December 2011 /Published online: 31 January 2012

(C) Dr. K C Chaudhuri Foundation 2012

The first edition of this book was published in 1994 and since then a new edition has been brought out regularly every $4-5$ y. Every edition has incorporated the newer advances that have taken place in the field of pediatric nephrology and the 5th edition is no exception. The updated, 26 chapters of this new edition deal with all the clinical problems encountered by the pediatrician and nephrologist. The new contributors with their vast experience, have done full justice to the chapters they have written. The basic format of the book remains the same $i$ e., devotion of first few chapters to anatomy and physiology which are important to understand before managing clinical problems of children with kidney diseases. The present edition also includes the key points in every chapter to highlight the important aspects. The text is supported by beautiful and self explanatory illustrations. The language is simple and easy to understand. The newer aspects which have been included in the book are so many that it is impossible to quote all of them. However the important ones are: newer modalities of treatment of nephrotic syndrome, concept of acute kidney injury along with biomarkers for its diagnosis, ambulatory blood pressure monitoring, inherited forms of renal tubular acidosis, continuous renal replacement therapy, drainage problems in peritoneal dialysis, disorders of micturition, fetal hydronephrosis, mineral bone disease and growth hormone therapy in chronic kidney disease, inherited forms of renal tubular acidosis, and tubulopathies. As suggested in my review of the 4th edition, a small section on prevention of kidney diseases has been added. Due to limited resources in our country, there should be guidelines for prevention and retardation of progression of chronic kidney disease.

The appendix, as important as any other part of the book, includes drug dosages, alkali and phosphate supplements, drug dosing in renal failure, renal length etc. and has added International Society of Peritoneal Dialysis 2010 guidelines for treatment of peritonitis in patients receiving peritoneal dialysis. A brief description of management of hypertensive urgency would have completed the discussion on hypertensive crisis. Overall this book is worth its value in gold and is a must companion for all those who care for children's renal problems.

P. K. Pruthi $(\bowtie)$

Pediatric Nephrologist, Department of Pediatrics,

Institute of Child Health, Sir Ganga Ram Hospital,

Rajinder Nagar,

New Delhi 110060, India

e-mail: pkpruthi@hotmail.com 